\title{
Measurement of the lifetime of a metastable excited state in $\mathrm{Bi}^{-}$
}

\author{
M. K. Kristiansson $\odot,{ }^{1,}{ }^{*}$ J. Karls, ${ }^{2}$ N. D. Gibson $\odot,{ }^{3}$ D. Hanstorp, ${ }^{2}$ H. T. Schmidt $\odot,{ }^{1}$ and C. W. Walter ${ }^{3}$ \\ ${ }^{1}$ Department of Physics, Stockholm University, Stockholm 10691, Sweden \\ ${ }^{2}$ Department of Physics, University of Gothenburg, Gothenburg 41296, Sweden \\ ${ }^{3}$ Department of Physics and Astronomy, Denison University, Granville, Ohio 43023, USA
}

(Received 12 November 2021; accepted 23 December 2021; published 18 January 2022)

\begin{abstract}
The lifetime of the ${ }^{3} P_{0}$ state of $\mathrm{Bi}^{-}$has been measured by selective photodetachment in a cryogenic ion-beam storage ring. By measuring the lifetime as a function of applied laser powers and extrapolating to zero laser power, a lifetime of $16.0 \pm 0.5 \mathrm{~s}$ is deduced for electric quadrupole decay of the excited state to the ground sate. The result provides a stringent test of recent state-of-the art theoretical calculations.
\end{abstract}

DOI: 10.1103/PhysRevA.105.L010801

Negative ions are important in a variety of physical situations, ranging from chemical reactions to planetary and stellar atmospheres to plasmas and discharges $[1,2]$. Since negative ions are not bound by a net Coulomb potential, correlation effects due to interactions of the electrons are relatively enhanced compared to neutral atoms and positive ions. Thus, negative ions present challenging but valuable opportunities to investigate electron correlation in atomic structure and dynamics, and they provide fertile testing grounds for stateof-the-art theoretical calculations [1,3-8].

The polarization potentials in negative ions are shallow and short ranged and can therefore only support one or at most a few bound states [1,5]. In most cases, any bound excited states are of the same parity as the ground state, so that electric dipole $(E 1)$ transitions are not allowed; indeed, $E 1$ transitions have only been observed to date in the four atomic negative ions $\mathrm{Os}^{-}$[9], $\mathrm{Ce}^{-}$[10], $\mathrm{La}^{-}$[11], and $\mathrm{Th}^{-}$ [12]. Transitions due to magnetic dipole $(M 1)$ and electric quadrupole $(E 2)$ interactions can occur between bound states of negative ions, but the transitions are weak and the lifetimes are consequently long (typically approximately seconds or greater) making experimental investigation very challenging. New capabilities to study such forbidden transitions have recently become available with the advent of new cryogenic storage ring facilities, such as the double electrostatic ion-ring experiment (DESIREE) [13-15] and the CSR [16,17] that can be used to measure decay rates of excited states of negative ions over unprecedentedly long time-scales of hours $[18,19]$.

The ground state of the bismuth negative ion is the $6 p^{4}{ }^{3} P_{2}$ level. The fine-structure levels are not ordered according to the $J$ quantum number and the only excited bound state has

\footnotetext{
*moa.kristiansson@fysik.su.se
}

Published by the American Physical Society under the terms of the Creative Commons Attribution 4.0 International license. Further distribution of this work must maintain attribution to the author(s) and the published article's title, journal citation, and DOI. Funded by Bibsam.
$J=0$ while the $J=1$ level is unbound [8]. This change in the ordering is a result of the strong relativistic effects present in a heavy atomic system such as $\mathrm{Bi}^{-}$. This leads to a breakdown of $L S$ coupling and strongly complicates the theoretical description of such systems. We chose to keep the $L S$-coupling notation throughout this Letter for compatibility with the literature, even though the states are heavily mixed. With $|\Delta J|=2$ and the same parity of both bound levels, the transition between them is an electric quadrupole (E2) transition. The observation of this transition by Walter et al. in 2021 was the first spectroscopic identification of an $E 2$ transition in an atomic negative ion [20], while a lifetime measurement of an excited level in $\mathrm{Pt}^{-}$decaying by an $E 2$ transition was performed in DESIREE in 2017 [21]. E2 interactions in negative ions provide particularly useful opportunities to gain insight into detailed electron correlation effects, as the transition rates depend crucially on the wave functions.

Figure 1 shows the energy level diagram of the bismuth negative ion. The electron affinity of bismuth, corresponding to the binding energy of the $\mathrm{Bi}^{-}\left(6 p^{4}{ }^{3} P_{2}\right)$ ground state relative to the $\mathrm{Bi}\left(6 p^{3}{ }^{4} S_{3 / 2}\right)$ ground state, is $942.369 \pm 0.013 \mathrm{meV}$ [22]. The binding energy of the ${ }^{3} P_{0}$ excited state was measured by Walter et al. to be $373.09 \pm 0.04 \mathrm{meV}$ using threshold photodetachment [20]. In the same study, the $\mathrm{Bi}^{-}{ }^{3} P_{2} \rightarrow{ }^{3} P_{0}$ $E 2$ transition energy was separately measured to be $569.27 \pm$ $0.03 \mathrm{meV}$ by a $(1+1)$ resonant photodetachment method [20].

In 2019, Su et al. made the first theoretical calculations of the $E 2$ radiative decay rate of the $\mathrm{Bi}^{-}{ }^{3} P_{0}$ excited state, obtaining a lifetime of $15.20 \mathrm{~s}$ (no uncertainty given) [8]. Recently as part of the 2021 study by Walter et al., Safronova and Cheung calculated the same $\mathrm{Bi}^{-}{ }^{3} P_{0}$ lifetime to be $16.5 \pm$ $0.7 \mathrm{~s}$ using the experimentally measured transition energy of $569.27 \pm 0.03 \mathrm{meV}$ [20]. While these two calculated lifetimes are fairly close, it is important to note that the quoted lifetime by $\mathrm{Su}$ et al. was obtained using their calculated transition energy for ${ }^{3} P_{2} \rightarrow{ }^{3} P_{0}$ of $624.0 \mathrm{meV}$, which is larger than the subsequently measured energy by $\sim 10 \%$ [20]. Since the $E 2$ lifetime scales inversely with transition energy to the fifth power [8], revising the lifetime by $\mathrm{Su}$ et al. using the measured energy would yield an adjusted lifetime of $24.05 \mathrm{~s}$, which is 


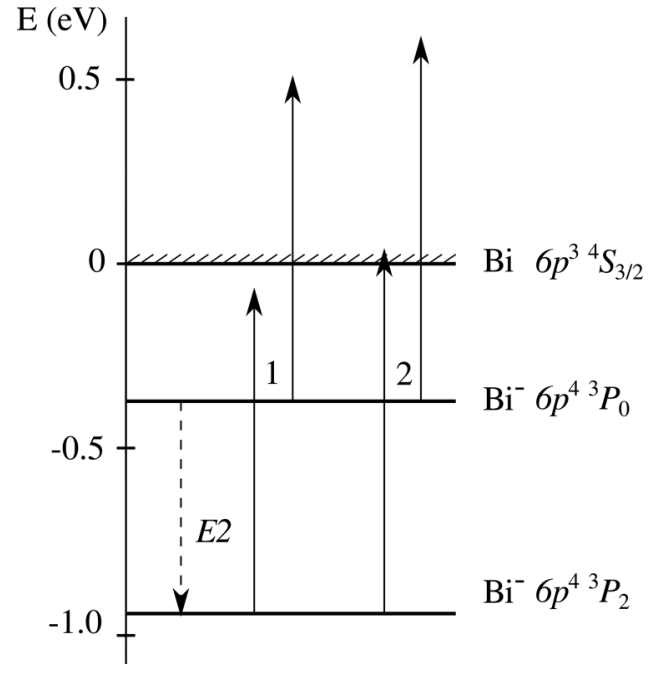

FIG. 1. Energy level diagram for $\mathrm{Bi}^{-}$showing $E 2$ radiative decay from the $\mathrm{Bi}^{-}{ }^{3} P_{0}$ excited state to the ${ }^{3} P_{2}$ ground state. The two photon energies that are used to photodetach the two bound states are indicated by the two sets of upward arrows. Arrow set 1 corresponds to $0.886 \mathrm{eV}(1400 \mathrm{~nm})$ and photodetaches only from the excited state. Arrow set 2 corresponds to $0.954 \mathrm{eV}(1300 \mathrm{~nm})$ and photodetaches from both the ground state and the excited state of $\mathrm{Bi}^{-}$. In the experiment, the time evolution of the $\mathrm{Bi}^{-}{ }^{3} P_{0}$ population in the storage ring is monitored to measure its lifetime by photodetaching either just the excited state or both states.

significantly longer than that predicted by the calculations of Walter et al. [20].

In the present study, we used state-selective photodetachment of $\mathrm{Bi}^{-}$ions in a storage ring to monitor the time evolution of both the ground and excited state populations. The extremely low pressure in the storage ring, coupled with the high sensitivity of the detection system, enables measurements of the ion populations over hundreds of seconds $[18,21,23]$ allowing precise observation of the weak $E 2$ radiative decay of the excited state. The results yield the first measured lifetime of the ${ }^{3} P_{0}$ state, providing a stringent test of the two different theoretically calculated lifetimes $[8,20]$ and giving new insights into the dynamics of this complex system.

The DESIREE at Stockholm University $[13,14]$ was used to store $\mathrm{Bi}^{-}$ions and through photodetachment, measure the lifetime of the excited state. DESIREE consists of two storage rings with a common straight section for studies of ion-ion collisions with sub-eV center-of-mass energies. The storage rings are contained in a vacuum chamber and kept at a temperature of $13.5 \pm 0.5 \mathrm{~K}$. The cryogenic temperature reduces the particle density of the rest gas in the vacuum chamber to a particle density of less than $10^{4} \mathrm{~cm}^{-3}$. $\mathrm{Bi}^{-}$ions are produced in a cesium sputter source where the high sputtering energy of several $\mathrm{keV}$ produces a relative population in the excited state that is many orders of magnitude larger than the Boltzmann factor at room temperature. The ions are injected into one of the storage rings and the excited state is allowed to equilibrate with the blackbody radiation of the chamber. This decay of the excited state is probed with photodetachment using light from a pulsed optical parametric oscillator laser system. The laser system has a repetition rate of $1 \mathrm{kHz}$ and a

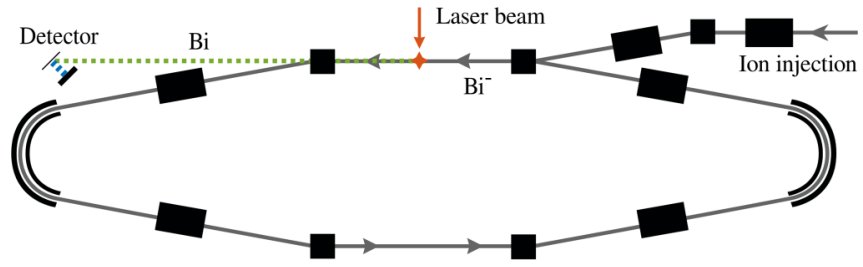

FIG. 2. Schematic of the ion storage ring used in the experiment. $\mathrm{Bi}^{-}$ions of $10 \mathrm{keV}$ energy are injected and stored in the ring. A transverse pulsed laser beam interacts with the ion beam. The neutral $\mathrm{Bi}$ atoms created in photodetachment travel straight forward and hit the micro-channel-plate detector.

pulse duration of $5 \mathrm{~ns}$. The two photon energies used are 0.886 and $0.954 \mathrm{eV}$ with varying average power from 0.2 to 11.5 $\mathrm{mW}$. The laser power is measured before the entrance window to the vacuum chamber. Due to divergence of the laser beam (the estimated diameter of the laser beam is about $1 \mathrm{~cm}$ ) and losses when passing through the vacuum chamber windows, the effective laser power interacting with the ions is at least a factor of 2 smaller than this. The laser light interacts with the ions in a transverse configuration in the straight section along the injection line on the storage ring according to Fig. 2 . Neutral atoms resulting from photodetachment are detected using a detector placed after the straight section. The detector consists of a glass plate covered by a single graphene layer for optimum surface conduction and secondary electron emission [24] followed by a microchannel plate triple-stack assembly. The signal resulting from photodetachment is collected during a time window of $10 \mu \mathrm{s}$, starting $16.7 \mu \mathrm{s}$ after the laser firing time, corresponding to the time of flight from the laser interaction region to the detector. This scheme results in a drastically reduced background from the detector (typically $\sim 3-5 \mathrm{~s}^{-1}$ ) and the ion-residual gas collisions. A second time window of $500 \mu$ s placed $130 \mu$ s after the signal gate is used to monitor and correct for the background signal.

In order to accurately measure the storage lifetime of an excited state, it is advantageous if the storage lifetime of the total ion beam is several times longer than the excited state lifetime. This is beneficial, since the decay of the excited state then can be distinguished from the decay of the ion beam. The lifetime of the ion beam was monitored by using a photon energy of $0.954 \mathrm{eV}$. This energy detaches both the ground state and the excited state corresponding to the arrows labeled 2 in Fig. 1. The storage lifetime was measured to be $1190 \pm 350 \mathrm{~s}$, which is much longer than the expected lifetime of the excited state. Another important factor is the ion-beam current; if a too high current is used the beam current has been found to decay faster than the characteristic exponential decay [14]. Using experience from dealing with ions of similar mass-to-charge ratio and energy, an ion-beam current of about $0.5 \mathrm{nA}$ (in the beginning of the measurement cycle) was used in order to avoid ion-ion interactions affecting the beam lifetime.

A photon energy of $0.886 \mathrm{eV}$ (arrow set 1 in Fig. 1) was used to probe the excited state as a function of storage time. This is shown in Fig. 3, where an average laser power of 8.2 $\mathrm{mW}$ is used. An exponential fit is made to extract the decay rate $\Gamma_{\text {tot }}$ which is a sum of several different effects according 


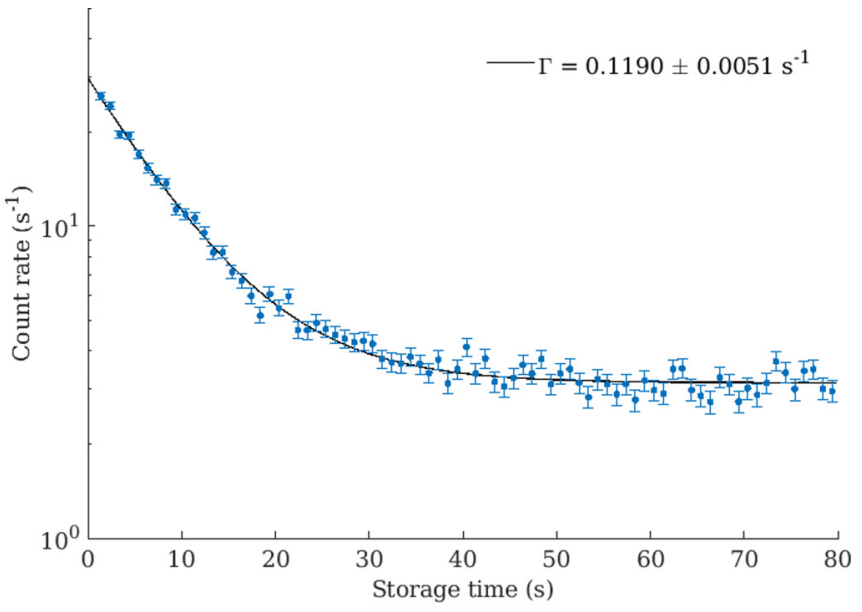

FIG. 3. Photodetachment signal as a function of storage time using a photon energy of $0.886 \mathrm{eV}$ and a laser power of $8.2 \mathrm{~mW}$. An exponential fit to the decay yields a decay rate of $0.1190 \pm$ $0.0051 \mathrm{~s}^{-1}$.

to

$$
\Gamma_{\text {tot }}=\Gamma_{0}+\Gamma_{\text {laser }}+\Gamma_{\text {other }}
$$

where $\Gamma_{0}$ is the radiative decay rate of the excited state, $\Gamma_{\text {laser }}$ is the decay rate due to the photodetachment process itself, and $\Gamma_{\text {other }}$ represents all other effects determining the undisturbed storage lifetime of the ion beam. $\Gamma_{\text {other }}$ can be taken into account by dividing the photodetachment signal by a fitted linear function to the background measured in between the laser pulses. Since the background mainly depends on the storage lifetime of the ion beam which is about $20 \mathrm{~min}$, a linear dependence is a good approximation of the exponential decay of the beam over the timescale of $80 \mathrm{~s}$ which is the time of a measurement cycle. In order to investigate the contribution of $\Gamma_{\text {laser }}$ to $\Gamma_{\text {tot }}$, a series of measurements were made where the laser power was varied. In Fig. 4 the decay rate is shown as a function of laser power. A linear decrease of the decay rate is expected with increasing laser power if only one-photon detachment is the process involved. A fit to a polynomial of first order $y=a x+b$ gives the unaffected radiative decay rate $b=\Gamma_{0}$ by extrapolation to zero laser power. This gives the radiative decay rate $\Gamma_{0}=0.0624 \pm 0.0017 \mathrm{~s}^{-1}$ corresponding to a lifetime of $\tau=\frac{1}{\Gamma_{0}}=16.0 \pm 0.5 \mathrm{~s}$.

The measured lifetime of $16.0 \pm 0.5 \mathrm{~s}$ is in excellent agreement with the previous calculation of $16.5 \pm 0.7 \mathrm{~s}$ by Walter et al. [20]. In that study the computational method was based on a combination of configuration interaction and the coupled-cluster method. However, when the experimental value is compared to the transition energy-scaled lifetime of $24.05 \mathrm{~s}$ by $\mathrm{Su}$ et al. [8] there is a significant difference. $\mathrm{Su}$ et al. used a fully relativistic multiconfiguration DiracHartree-Fock method to investigate structural effects for a

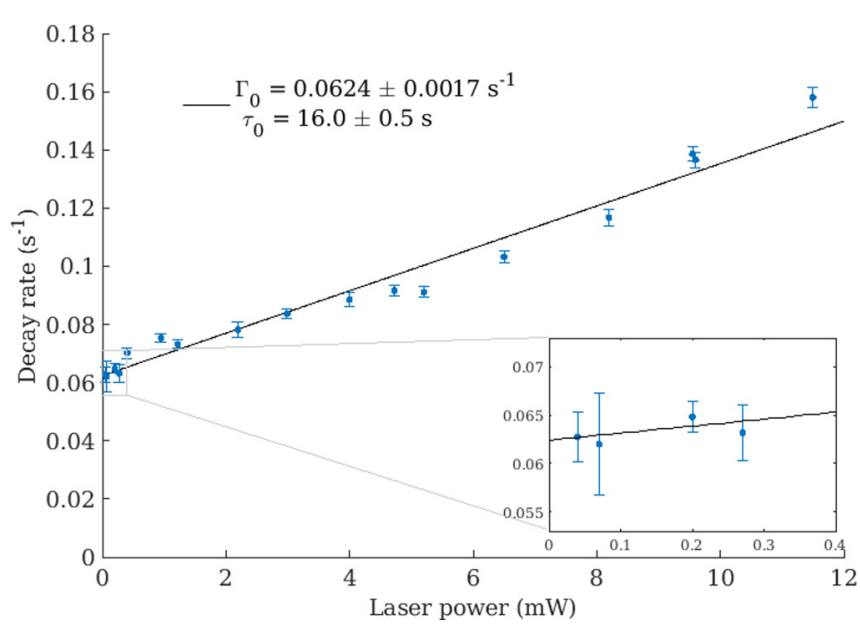

FIG. 4. The measured decay rate as a function of average laser power going from 0.02 to $11.5 \mathrm{~mW}$. A first-order polynomial is fitted to the data in order to extract the radiative decay rate $\Gamma_{0}$ which yields $\Gamma_{0}=0.0624 \pm 0.0017 \mathrm{~s}^{-1}$. This gives a lifetime of $\tau_{0}=\frac{1}{\Gamma_{0}}=$ $16.0 \pm 0.5 \mathrm{~s}$. The inset in the bottom right shows a zoomed-in view in the low power region, close to the intersection with the decay-rate axis.

series of negative ions homologous to $\mathrm{N}^{-}$, including $\mathrm{Bi}^{-}$. The two calculations were performed using different computational methods and different procedures and this illustrates the importance of experiments like the present in order to further understand these complex systems with strong correlation effects. Accurate lifetime measurements can be of assistance when comparing computational results in order to further improve the accuracy of the computational methods. In order to conclude if one computational method is preferred to the other, more similar strategic approaches would have to be applied in order to compare the two methods. We can, however, conclude that for this case the computational result of Walter et al. [20] gives better agreement with the experiment than the result of $\mathrm{Su}$ et al. [8].

The present result is an illustrative example of the importance of the exceptional temperature and pressure conditions obtained in a cryogenic experiment such as the DESIREE. These conditions are the decisive factor for measuring high precision lifetimes of the long-lived excited states in negative atomic ions.

We thank the technical staff and operators of the DESIREE facility for their contributions to this work. We thank F. Vassallo for contributions to the data analysis. This work was performed at the Swedish National Research Infrastructure, DESIREE (Swedish Research Council Contract No. 201700621). H.T.S. and D.H. acknowledge the Swedish Research Council for individual project grants (with Contracts No. 2018-04092, No. 2016-03650, and No. 2020-03505.). N.D.G. and C.W.W. were supported, in part, by US National Science Foundation (NSF) Grants No. PHY-1707743 and No. PHY2110444.
[1] D. J. Pegg, Structure and dynamics of negative ions, Rep. Prog. Phys. 67, 857 (2004).
[2] T. J. Millar, C. Walsh, and T. A. Field, Negative ions in space, Chem. Rev. 117, 1765 (2017). 
[3] C. F. Fischer, Correlation in negative ions, Phys. Scr. 40, 25 (1989).

[4] T. Andersen, H. K. Haugen, and H. Hotop, Binding energies in atomic negative ions: III, J. Phys. Chem. Ref. Data 28, 1511 (1999).

[5] T. Andersen, Atomic negative ions: Structure, dynamics and collisions, Phys. Rep. 394, 157 (2004).

[6] V. K. Ivanov, Theoretical studies of photodetachment, Radiat. Phys. Chem. 70, 345 (2004).

[7] M. T. Eiles and C. H. Greene, Extreme Correlation and Repulsive Interactions in Highly Excited Atomic Alkali Anions, Phys. Rev. Lett. 121, 133401 (2018).

[8] Y. Su, R. Si, K. Yao, and T. Brage, The structure and radiative lifetimes of negative ions homologous to $\mathrm{N}^{-}$, J. Phys. B: At., Mol. Opt. Phys. 52, 125002 (2019).

[9] R. C. Bilodeau and H. K. Haugen, Experimental Studies of $\mathrm{Os}^{-}$: Observation of a Bound-Bound Electric Dipole Transition in an Atomic Negative Ion, Phys. Rev. Lett. 85, 534 (2000).

[10] C. W. Walter, N. D. Gibson, Y.-G. Li, D. J. Matyas, R. M. Alton, S. E. Lou, R. L. Field, D. Hanstorp, L. Pan, and D. R. Beck, Experimental and theoretical study of bound and quasibound states of $\mathrm{Ce}^{-}$, Phys. Rev. A 84, 032514 (2011).

[11] C. W. Walter, N. D. Gibson, D. J. Matyas, C. Crocker, K. A. Dungan, B. R. Matola, and J. Rohlén, Candidate for Laser Cooling of a Negative Ion: Observations of Bound-Bound Transitions in $\mathrm{La}^{-}$, Phys. Rev. Lett. 113, 063001 (2014).

[12] R. Tang, R. Si, Z. Fei, X. Fu, Y. Lu, T. Brage, H. Liu, C. Chen, and C. Ning, Observation of electric-dipole transitions in the laser-cooling candidate $\mathrm{Th}^{-}$and its application for cooling antiprotons, Phys. Rev. A 103, 042817 (2021).

[13] R. D. Thomas, H. T. Schmidt, G. Andler, M. Björkhage, M. Blom, L. Brännholm, E. Bäckström, H. Danared, S. Das, N. Haag, P. Halldén, F. Hellberg, A. I. S. Holm, H. A. B. Johansson, A. Källberg, G. Källersjö, M. Larsson, S. Leontein, L. Liljeby, P. Löfgren et al., The double electrostatic ion ring experiment: A unique cryogenic electrostatic storage ring for merged ion-beams studies, Rev. Sci. Instrum. 82, 065112 (2011).

[14] H. T. Schmidt, R. D. Thomas, M. Gatchell, S. Rosén, P. Reinhed, P. Löfgren, L. Brännholm, M. Blom, M. Björkhage, E. Bäckström, J. D. Alexander, S. Leontein, D. Hanstorp, H. Zettergren, L. Liljeby, A. Källberg, A. Simonsson, F. Hellberg, S. Mannervik, M. Larsson et al., First storage of ion beams in the double electrostatic ion-ring experiment: DESIREE, Rev. Sci. Instrum. 84, 055115 (2013).

[15] H. T. Schmidt, G. Eklund, K. C. Chartkunchand, E. K. Anderson, M. Kamińska, N. de Ruette, R. D. Thomas, M. K. Kristiansson, M. Gatchell, P. Reinhed, S. Rosén, A. Simonsson, A. Källberg, P. Löfgren, S. Mannervik, H. Zettergren, and H. Cederquist, Rotationally Cold $\mathrm{OH}^{-}$Ions in the Cryogenic
Electrostatic Ion-Beam Storage Ring DESIREE, Phys. Rev. Lett. 119, 073001 (2017).

[16] R. von Hahn, A. Becker, F. Berg, K. Blaum, C. Breitenfeldt, H. Fadil, F. Fellenberger, M. Froese, S. George, J. Goeck, M. Grieser, F. Grussie, E. A. Guerin, O. Heber, P. Herwig, J. Karthein, C. Krantz, H. Kreckel, M. Lange, F. Laux et al., The cryogenic storage ring CSR, Rev. Sci. Instrum. 87, 063115 (2016).

[17] C. Meyer, A. Becker, K. Blaum, C. Breitenfeldt, S. George, J. Göck, M. Grieser, F. Grussie, E. A. Guerin, R. von Hahn, P. Herwig, C. Krantz, H. Kreckel, J. Lion, S. Lohmann, P. M. Mishra, O. Novotný, A. P. O'Connor, R. Repnow, S. Saurabh et al., Radiative Rotational Lifetimes and State-Resolved Relative Detachment Cross Sections from Photodetachment Thermometry of Molecular Anions in a Cryogenic Storage Ring, Phys. Rev. Lett. 119, 023202 (2017).

[18] E. Bäckström, D. Hanstorp, O. M. Hole, M. Kaminska, R. F. Nascimento, M. Blom, M. Björkhage, A. Källberg, P. Löfgren, P. Reinhed, S. Rosén, A. Simonsson, R. D. Thomas, S. Mannervik, H. T. Schmidt, and H. Cederquist, Storing keV Negative Ions for an Hour: The Lifetime of the Metastable ${ }^{2} P_{1 / 2}^{o}$ level in ${ }^{32} \mathrm{~S}^{-}$, Phys. Rev. Lett. 114, 143003 (2015).

[19] D. Müll, F. Grussie, K. Blaum, S. George, J. Göck, M. Grieser, R. von Hahn, Z. Harman, A. Kálosi, C. H. Keitel, C. Krantz, C. Lyu, O. Novotný, F. Nuesslein, D. Paul, V. C. Schmidt, S. Singh, S. Sunil Kumar, X. Urbain, A. Wolf et al., Metastable states of $\mathrm{Si}^{-}$observed in a cryogenic storage ring, Phys. Rev. A 104, 032811 (2021).

[20] C. W. Walter, S. E. Spielman, R. Ponce, N. D. Gibson, J. N. Yukich, C. Cheung, and M. S. Safronova, Observation of an Electric Quadrupole Transition in a Negative Ion: Experiment and Theory, Phys. Rev. Lett. 126, 083001 (2021).

[21] K. C. Chartkunchand, M. Kamińska, E. K. Anderson, M. K. Kristiansson, G. Eklund, O. M. Hole, R. F. Nascimento, M. Blom, M. Björkhage, A. Källberg, P. Löfgren, P. Reinhed, S. Rosén, A. Simonsson, R. D. Thomas, S. Mannervik, V. T. Davis, P. A. Neill, J. S. Thompson, D. Hanstorp et al., Radiative lifetimes of the bound excited states of $\mathrm{Pt}^{-}$, Phys. Rev. A 94, 032501 (2016).

[22] R. C. Bilodeau and H. K. Haugen, Electron affinity of Bi using infrared laser photodetachment spectroscopy, Phys. Rev. A 64, 024501 (2001).

[23] M. K. Kristiansson, S. Schiffmann, J. Grumer, J. Karls, N. de Ruette, G. Eklund, V. Ideböhn, N. D. Gibson, T. Brage, H. Zettergren, D. Hanstorp, and H. T. Schmidt, Experimental and theoretical studies of excited states in $\mathrm{Ir}^{-}$, Phys. Rev. A 103, 062806 (2021).

[24] J. Warbinek, D. Leimbach, D. Lu, K. Wendt, D. J. Pegg, A. Yurgens, D. Hanstorp, and J. Welander, A graphene-based neutral particle detector, Appl. Phys. Lett. 114, 061902 (2019). 